\title{
Schistosoma mansoni: ação da lovastatina no modelo murino
}

\author{
Schistosoma mansoni: the action of lovastatin on the murine model
}

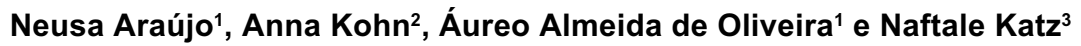

\begin{abstract}
Resumo Com o objetivo de avaliar a ação da lovastatina na postura do Schistosoma mansoni foram usados camundongos infectados com $100 \pm 10$ cercárias da cepa $L E$. Trinta dias após a infecção, os animais foram tratados com 100, 200 e 400mg/kg de lovastatina, via oral, durante cinco dias consecutivos e sacrificados 7,15,30 ou 60 dias após o tratamento. Foram analisados: distribuição de vermes no mesentério e fígado, mortalidade de vermes no fígado, alteração do oograma, contagem de ovos no jejuno e fígado, presença de ovos intra-uterino e morfologia dos vermes dos grupos tratados e controle (animais infectados e não tratados). Diferenças estatisticamente significativas foram encontradas, na dose de $400 \mathrm{mg} / \mathrm{kg}$, entre grupos tratados e controles quando se considerou a presença de ovos no útero, a alteração do oograma, ovos nos diferentes estágios de desenvolvimento no jejuno e fígado e no comprimento do corpo dos vermes machos e fêmeas. $O$ estudo morfológico dos vermes mostrou alterações degenerativas sendo as principais no aparelho reprodutor, com redução e alteração dos folículos vitelínicos e do ovário das fêmeas e modificações nos testículos dos machos. Os resultados apresentados levam à conclusão que a droga em estudo reduz, consideravelmente, a oviposição das fêmeas do S. mansoni, aumenta o tamanho dos vermes, provoca alterações no sistema reprodutivo de machos e fêmeas e pode ocasionar morte de parte significativa da população de vermes na dose de 400mg/kg.
\end{abstract}

Palavras-chaves: Schistosoma mansoni. Lovastatina. HMG-CoA redutase.

Abstract Aimed to evaluate the lovastatin action on Schistosoma mansoni oviposition, infected mice with $100 \pm 10$ cercariae of the LE strain were used. Thirty days after infection the animals were treated with 100, 200 and $400 \mathrm{mg} / \mathrm{kg}$ of lovastatin, per os, during five consecutive days and then sacrificed 7, 15, 30 or 60 days after treatment. We analyzed: distribution of worms in mesenteries and liver; mortality of worms in the liver; alteration of the oogram; eggs counting in the jejunum and liver; presence of intrauterine eggs and morphology of the worms from the treated and control groups (infected and not treated animals). Significant statistical differences were found between treated and control groups when the presence of intrauterine eggs was considered and also, alteration on the oogram, eggs at different stages of development in jejunum, liver and in the body length of males and females. The morphological study of the worms showed that the degenerative modifications occurred, mainly in the reproductive system, with reduction and alteration of the viteline follicles and the ovary of the females. Also modifications in the males testicles were observed. The results suggest that the drug under study reduces, considerably, the female S. mansoni, oviposition increases the worms' size, leads to alteration in the reproductive system of males and females. It also may induce death of a significant part of the worm populations at the dose of $400 \mathrm{mg} / \mathrm{kg}$.

Key-words: Schistosoma mansoni. Lovastatina. HMG-CoA reductase.

Em 1967, Campbell e Cuckler mostraram que camundongos infectados pelo Schistosoma mansoni tiveram a produção de ovos suprimida pelo tratamento com nicarbazin (complexo equimolar de 1,1dinitrocarbanilida e 2-hidroxi-1,6-dimetilpirimidina). A inclusão de $0,2 \%$ de nicarbazin na dieta dos animais, inibiu completamente a postura de ovos, sem entretanto matar os vermes. A inibição foi reversível, com as fêmeas reiniciando a postura quando o tratamento foi interrompido.

Em 1969, Pellegrino \& Katz mostraram que nicarbazin incorporado à dieta de camundongos infectados experimentalmente pelo $S$. mansoni, na proporção de 1e 1,5\% interrompeu a postura de ovos.

\footnotetext{
1. Centro de Pesquisas René Rachou da Fundação Oswaldo Cruz, Belo Horizonte, MG. 2. Departamento de Helmintologia do Instituto Oswaldo Cruz da Fundação Oswaldo Cruz, Rio de Janeiro, RJ. 3. Centro de Pesquisas René Rachou da Fundação Oswaldo Cruz, Belo Horizonte, MG e CNPq. Endereço para correspondência: Dra. Neusa Araújo. Centro de Pesquisas René Rachou/FIOCRUZ. Av. Augusto de Lima 1715, 30190-002, Barro Preto, Belo Horizonte, MG, Brasil.

Tel: 5531 3295-3566, Fax: 5531 3295-3115

e-mail: araujon@cpqrr.fiocruz.br

Recebido para publicação em 11/1/2001.
} 
Na proporção de $0,5 \%$ houve interrupção da postura em $50 \%$ dos animais examinados. O efeito da droga cessou assim que houve interrupção do tratamento. Quando foram usados hamsters, não foi observada interrupção da postura quando o nicarbazin foi incorporado à dieta (1\%) por 36 dias ou $2 \%$ em dois tratamentos de cinco dias consecutivos separados por dois dias de intervalo. Macacos Cebus infectados experimentalmente com $S$. mansoni e tratados com nicarbazin, mostraram interrupção temporária da postura observada através de curetagem retal.

Estes mesmos autores, observaram que a diaminidifenilsulfona (DDS) foi capaz de interromper a postura do S. mansoni em camundongos, hamsters e macacos Cebus. A dose de 0,05\% de DDS incorporada à dieta dos camundongos apresentou atividade supressiva da oviposição, atividade esta que foi transitória, desaparecendo imediatamente após a suspensão da droga ${ }^{6}$. Em 1987, Vandewaa \& Bennett mostraram que a substância mevinolin, um inibidor da síntese de colesterol, bloqueava a produção de ovos de S. mansoni. Chen e cols, em 1990 mostraram que a administração de $0,2 \%$ de mevinolin na dieta de camundongos infectados pelo S. mansoni durante 14 dias resultou em $96-100 \%$ de eliminação de parasitas adultos; a administração da mesma dose por dois dias antes e 15 dias depois da infecção resultou em 93-96\% de redução dos parasitas adultos.

Neste trabalho são apresentados os resultados obtidos em camundongos infectados experimentalmente pelo $S$. mansoni e tratados com lovastatina, medicamento usado na clínica médica para redução dos níveis de colesterol.

\section{MATERIAL E MÉTODOS}

Terapêutica experimental. Foram usados camundongos albinos (peso médio 20 gramas) infectados por via subcutânea com $100 \pm 10$ cercárias da cepa LE de S. mansoni. Após 30 dias da infecção, os animais foram tratados, por via oral, com lovastatina (Mevacor®, Merck Sharp \& Dohme) nas doses de 100, 200 ou $400 \mathrm{mg} / \mathrm{kg}$, durante cinco dias consecutivos. Os animais foram sacrificados por fratura cervical e perfundidos 7 , 15,30 ou 60 dias após o término do tratamento. Os mesmos procedimentos foram desenvolvidos em grupos controles não tratados com a droga conforme metodologia descrita, em 1968, por Pellegrino \& Katz 4 .

Avaliação de atividade. A atividade da droga foi avaliada através das percentagens de distribuição dos vermes no mesentério e fígado, dos vermes mortos no fígado e da alteração do oograma. Determinou-se também a percentagem de fêmeas que apresentavam ovos intra-uterino, e de ovos nos diversos estágios, através da observação do oograma ${ }^{5}$. Foi feita a recuperação e contagem do número de ovos de S. mansoni após digestão do fígado e do jejuno de cada animal dos grupos tratados e controle e os resultados foram expressos em número médio de ovos por grama de tecido ${ }^{7}$.

Estudo morfológico dos vermes. Os vermes recuperados por perfusão foram fixados com formol neutro a $10 \%$, corados pelo carmim clorídrico alcoólico, desidratados na série alcoólica, diafanizados com creosoto de Faia e montados em bálsamo do Canadá. As alterações morfológicas do sistema reprodutivo foram observadas com aumento de $400 X^{3}$.

Apresentação e análise dos dados. Os dados foram apresentados como média, percentagem ou média \pm desvio padrão. As médias foram comparadas pelo teste $\mathrm{t}$ de Student ou por análise de variância, sendo estipulado o nível de significância de $p=0,05^{8}$.

\section{RESULTADOS}

$\mathrm{Na}$ Tabela 1 podem ser vistos os resultados obtidos em camundongos infectados experimentalmente pela cepa LE de S. mansoni, tratados com lovastatina no
30 ำ dia da infecção e sacrificados 30 dias após o término do tratamento. As doses utilizadas foram de 100, 200 ou $400 \mathrm{mg} / \mathrm{kg}$, doses únicas ou administradas durante

Tabela 1 - Resultados obtidos em camundongos infectados experimentalmente pela cepa LE de Schistosoma mansoni, tratados com lovastatina, via oral 30 dias após a infecção e sacrificados 30 dias após o término do tratamento.

\begin{tabular}{|c|c|c|c|c|c|c|c|}
\hline \multirow{2}{*}{$\begin{array}{l}\text { Esquema de tratamento } \\
\mathrm{mg} / \mathrm{kg} / \text { dia/dias }\end{array}$} & \multirow{2}{*}{$\begin{array}{c}\text { Animais } \\
\text { examinados } n^{\circ}\end{array}$} & \multirow{2}{*}{$\begin{array}{r}\text { Média de } \\
\text { vermes }\end{array}$} & \multicolumn{2}{|c|}{ Distribuição de vermes \% } & \multirow{2}{*}{$\begin{array}{c}\text { Vermes mortos } \\
\text { no fígado } \%\end{array}$} & \multirow{2}{*}{$\begin{array}{c}\text { Presença de ovos } \\
\text { no útero \% }\end{array}$} & \multirow{2}{*}{$\begin{array}{r}\text { Alteração do } \\
\text { oograma \% }\end{array}$} \\
\hline & & & mesentério & fígado & & & \\
\hline$\overline{100 / 5}$ & 2 & 27,5 & 83,6 & 16,4 & 10,9 & 37,5 & 0,0 \\
\hline $200 / 5$ & 7 & 20,6 & 91,0 & 9,0 & 5,6 & 53,3 & 28,6 \\
\hline 400/1 & 5 & 17,6 & 85,2 & 14,8 & 14,8 & $16,7^{*}$ & 20,0 \\
\hline $400 / 5$ & 7 & 17,7 & 67,7 & 32,3 & 21,8 & 37,8 & $42,9^{*}$ \\
\hline Controle & 10 & 19,8 & 84,3 & 15,7 & 0,0 & 67,0 & 0,0 \\
\hline
\end{tabular}

${ }^{*} p \leq 0,05$

cinco dias consecutivos. A dose de $400 \mathrm{mg} / \mathrm{kg} / \mathrm{dia}$ administrada por cinco dias consecutivos foi a que apresentou maior mortalidade dos vermes $(21,8 \%)$ e o maior percentual de alteração do oograma (42,9\%).
A Tabela 2 mostra os resultados obtidos em camundongos infectados com cercárias da cepa LE de S. mansoni e tratados com lovastatina na dose de $400 \mathrm{mg} / \mathrm{kg}$ x 5 dias consecutivos, por via oral 30 dias 
Tabela 2 - Resultados obtidos em camundongos infectados experimentalmente pela cepa LE de Schistosoma mansoni tratados com lovastatina, $400 \mathrm{mg} / \mathrm{kg} /$ dia x 5 dias consecutivos, por via oral 30 dias após a infecção e sacrificados 7, 15, 30 ou 60 dias após o término do tratamento.

\begin{tabular}{|c|c|c|c|c|c|c|c|c|}
\hline \multirow{2}{*}{$\begin{array}{l}\text { Examinados após } \\
\text { de tratamento }\end{array}$} & \multicolumn{2}{|c|}{ Animais $\mathrm{n}^{\circ}$} & \multirow{2}{*}{$\begin{array}{c}\text { Média de } \\
\text { vermes }\end{array}$} & \multicolumn{2}{|c|}{ Distribuição de vermes \% } & \multirow{2}{*}{$\begin{array}{c}\text { Vermes mortos } \\
\text { no fígado } \%\end{array}$} & \multirow{2}{*}{$\begin{array}{c}\text { Presença de ovos } \\
\text { no útero \% }\end{array}$} & \multirow{2}{*}{$\begin{array}{r}\text { Alteração do } \\
\text { oograma \% }\end{array}$} \\
\hline & tratados & examinados & & mesentério & fígado & & & \\
\hline 7 & 10 & 10 & 22,2 & 84,2 & 15,8 & 2,7 & $33,9^{*}$ & 10,0 \\
\hline 15 & 10 & 5 & 22,4 & 83,9 & 16,1 & 12,5 & $38,8^{*}$ & $60,0^{*}$ \\
\hline 30 & 10 & 9 & 15,8 & $32,4^{*}$ & $67,6^{*}$ & $28,9^{*}$ & $40,4^{*}$ & $77,8^{*}$ \\
\hline 60 & 10 & 6 & 25,8 & 79,9 & 20,1 & 9,8 & $32,9^{*}$ & $83,3^{*}$ \\
\hline Controle & - & 10 & 29,5 & 88,1 & 11,9 & 0,0 & 67,2 & 0,0 \\
\hline
\end{tabular}

${ }^{*} \mathrm{p} \leq 0,05$

após a infecção e sacrificados 7, 15, 30 ou 60 dias após o término do tratamento. Em relação à distribuição de vermes no mesentério e fígado, somente o grupo sacrificado 30 dias após o tratamento apresentou deslocamento significativo dos vermes para o fígado. Vermes mortos no fígado foram encontrados em todos os grupos tratados variando de 2,7 a $28,9 \%$ nos diferentes dias de observação. Todos os grupos mostraram resultados significativos quando comparados ao controle, em relação à presença de ovos intrauterino e alteração do oograma, excetuando-se neste último parâmetro o grupo sacrificado sete dias após o tratamento.
Nos grupos tratados com a dose de $400 \mathrm{mg} / \mathrm{kg} / \mathrm{dia}$ durante cinco dias a percentagem de ovos nos diferentes estágios no jejuno diferiu significativamente do grupo controle, mostrando um grande aumento do número de ovos mortos e diminuição acentuada dos ovos viáveis dos estágios iniciais.

Também foi observada diminuição significativa da média do número de ovos por grama de fígado, quando comparado ao grupo controle (Tabela 3).

A avaliação biométrica dos vermes demonstrou aumento significativo no tamanho do comprimento total do corpo dos vermes tratados, principalmente da fêmeas quando comparado com o grupo controle (Tabela 4).

\begin{tabular}{|c|c|c|c|c|c|c|c|}
\hline \multirow{2}{*}{$\begin{array}{l}\text { Dias após } \\
\text { o tratamento }\end{array}$} & \multicolumn{6}{|c|}{ Média de ovos nos diferentes estágios de desenvolvimento no jejuno (\%) } & \multirow{2}{*}{$\begin{array}{l}\text { Média do } \mathrm{n}^{\circ} \text { de ovos } \\
\text { por grama fígado }\end{array}$} \\
\hline & $1^{\circ}$ & $2^{\circ}$ & $3^{\circ}$ & $4^{\circ}$ & maduro & morto & \\
\hline 7 & $5,7^{*}$ & $7,5^{*}$ & 8,3 & 9,5 & 23,4 & $45,8^{*}$ & $133,7^{*}$ \\
\hline 15 & $1,1^{*}$ & $1,3^{*}$ & $3,2^{*}$ & 8,8 & $59,0^{*}$ & 26,6 & $167,7^{*}$ \\
\hline 30 & $0,2^{*}$ & $0,4^{*}$ & $0,6^{*}$ & $3,2^{*}$ & $41,2^{*}$ & $54,4^{*}$ & $112,5^{*}$ \\
\hline 60 & $1,4^{*}$ & $1,4^{*}$ & $0,5^{*}$ & $4,1^{*}$ & $45,6^{*}$ & $47,0^{*}$ & $41,8^{*}$ \\
\hline Controle & 20,0 & 17,9 & 13,5 & 15,4 & 17,2 & 16,0 & $223,6^{*}$ \\
\hline
\end{tabular}

${ }^{*} p \leq 0,05$

Tabela 4 - Variação do comprimento do corpo de Schistosoma mansoni em camundongos tratados com lovastatina $400 \mathrm{mg} / \mathrm{kg}$ durante 5 dias consecutivos.

\begin{tabular}{|c|c|c|c|c|c|c|}
\hline \multirow{2}{*}{$\begin{array}{l}\text { Dias após } \\
\text { o tratamento }\end{array}$} & \multicolumn{3}{|c|}{ Comprimento do corpo (vermes machos)* } & \multicolumn{3}{|c|}{ Comprimento do corpo (vermes fêmeas) ${ }^{\star}$} \\
\hline & mínimo & máximo & média \pm desvio Padrão & mínimo & máximo & média \pm desvio padrão \\
\hline 7 & $7,1^{* *}$ & 11,4 & $9,4 \pm 1,1^{\star *}(30)$ & $8,0^{* *}$ & $13,8^{* *}$ & $11,0 \pm 1,4^{* *}(25)$ \\
\hline Controle & 4,0 & 8,8 & $6,3 \pm 1,531$ & 4,4 & 7,9 & $6,4 \pm 0,9(24)$ \\
\hline 30 & $7,5^{* *}$ & 11,0 & $8,3 \pm 1,4(11)$ & $11,9^{* *}$ & 14,0 & $12,6 \pm 1,5^{\star *}(09)$ \\
\hline Controle & 5,0 & 9,4 & $7,2 \pm 1,414$ & 8,0 & 11,4 & $9,2 \pm 1,0(11)$ \\
\hline $60^{* * *}$ & 6,6 & 14,4 & $9,4 \pm 1,918$ & 10,8 & 20,4 & $12,4 \pm 3,2(13)$ \\
\hline
\end{tabular}

* em milímetros

** $\mathrm{p} \leq 0,05$

${ }^{* * *}$ Após 60 dias não havia animal sobrevivente no grupo controle

( ) - Número de medidas realizadas

O estudo morfológico dos vermes machos e fêmeas coletados de camundongos sacrificados sete dias após o término do tratamento com lovastatina, $400 \mathrm{mg} / \mathrm{kg} \times 5$ dias mostrou alterações degenerativas no aparelho reprodutor, com redução e alteração dos folículos vitelínicos e do ovário das fêmeas e modificações nos testículos dos machos. O conteúdo intestinal das fêmeas exibiu acentuada despigmentação, $\mathrm{O}$ tegumento e o parênquima apresentaram-se edemaciados e nos machos os tubérculos apresentaram distribuição irregular. Após 30 dias de tratamento, a maior parte dos vermes apresentava aspecto normal, e depois de 90 dias pequena parte dos vermes ainda apresentavam alterações à nível de parênquima e no tegumento e tubérculo dos machos e somente alguns exemplares fêmeas apresentavam alterações no aparelho reprodutor e despigmentação parcial ou total do conteúdo intestinal. 


\section{DISCUSSÃO}

Algumas drogas produzem a interrupção específica da postura de ovos de S. mansoni ${ }^{256}$ e neste estudo foi avaliada a lovastatina que também reduz, consideravelmente a oviposição pelas fêmeas do parasita, podendo, inclusive, ocasionar a morte de alguns vermes.

Resultados apresentados por Vandewaa et al ${ }^{10}$ trabalhando com mevinolato, farnesol, e mevinolin in vivo e in vitro, sugerem que a produção de ovos na esquistossomose está associada com a atividade da enzima HMG-CoA redutase e que lípides não esteróis, produzidos no metabolismo bioquímico, regulados por esta enzima estimulam a produção de ovos. Chen et $\mathrm{al}^{2}$ trabalhando com camundongos infectados com S. mansoni e tratados com baixas doses de mevinolin, observaram a parada de postura pelo parasita e o bloqueio da patologia associada à infecção. A administração de $0,2 \%$ de mevinolin na dieta de camundongos infectados por 14 dias, resultou em $96 \%$ de eliminação dos parasitas. A mesma dose administrada por dois dias antes e 15 dias após a infecção resultou em 93-95\% de redução do número de parasitas adultos. A administração de mevinolin com $0,5 \%$ de mevalonato bloqueou a atividade antiesquistossomicida do mevinolin, sugerindo que mevalonato e/ou metabolitos não somente são de vital importância para a produção de ovos em S. mansoni, como também para a sobrevivência do parasita.

A lovastatina é uma lactona, forma inativa do ácido hidroxílico aberto correspondente, é um potente inibidor de síntese endógena de colesterol. Após a absorção gastrointestinal a lovastatina é rapidamente hidrolisada para o hidroxiácido aberto um inibidor competitivo da 3- hidroxi 3- metilglutaril Coenzima A (HMG-CoA) redutase, enzima que catalisa uma etapa precoce e limitante na biossíntese do colesterol. Em estudos clínicos a lovastatina reduziu as concentrações de colesterol plasmático total e lipoproteína de baixa densidade (LDL) e a lipoproteína de densidade muito baixa (VLDL) ligadas ao colesterol.

No presente trabalho, a análise do conjunto de parâmetros mostrou que apesar da percentagem de mortalidade dos vermes ser relativamente baixa (em torno de $30 \%$ ) a administração de lovastatina nos esquemas terapêuticos apresentados, reduziu significativamente a oviposição pelas fêmeas do $S$. mansoni apresentando alterações de oograma em até $80 \%$ dos camundongos e com média de ovos no jejuno e fígado significativamente menor nos animais quando comparados com o grupo controle. A análise morfológica dos vermes mostrou alterações degenerativas, principalmente no aparelho reprodutor dos vermes com redução e alteração dos folículos vitelínicos e do ovário das fêmeas e modificações nos testículos dos machos.

A ação da lovastatina na postura do $S$. mansoni pode ser vista inicialmente pela diminuição da presença de ovo no útero, associada à alteração dos vermes machos e fêmeas e resultando na diminuição da postura e consequentemente na alteração do oograma.

É interessante salientar o aumento significativo no tamanho dos vermes machos e fêmeas dos grupos tratados em relação ao tamanho dos vermes dos grupos controles.

Sugere-se que estudos sejam realizados visando o acompanhamento clínico e parasitológico de pacientes hipercolesterotêmicos e que tenham esquistossomose associada para avaliação da atividade da lovastatina.

\section{REFERÊNCIAS BIBLIOGRÁFICAS}

1. Campbell WC, Cuckler AC. Inibition off egg production of Schistosoma mansoni in mice treated with nicarbazin. Journal of Parasitology 53: 977-980, 1967.

2. Chen GZ, Foster L, Bennett JL. Antischistosomal action of mevinolin evidence that 3-hydroxi-methylglutaryl-coenzime a reductase activity Schistosoma mansoni is vital for parasite survival. Naunin-Schmiedebergs-Archives-Pharmacolology 342: 477-482, 1990.

3. Kohn A, Serapião CJ, Katz N, Dias EP. Ação da oxamniquine sobre o Schistosoma mansoni em camundongos experimentalmente infectados. Revista do Instituto Medicina Tropical de São Paulo 21: 217-227, 1979.

4. Pellegrino J, Katz N. Experimental chemotherapy of Schistosomiasis mansoni. Advance in Parasitology 6:233-290, 1968.

5. Pellegrino J, Katz N. Experimental chemotherapy of Schistosomiasis. IV. Oogram studies with nicarbazin, an egg- supressive agent. Revista do Instituto de Medicina Tropical de São Paulo 11: 215-221, 1969.

6. Pellegrino J, Katz $\mathrm{N}$. Terapêutica experimental da esquistossomose. $X$ - Ação da diaminofenilsulfona (DDS) na esquistossomose experimental. Revista do Instituto de Medicina Tropical de São Paulo 17: 199-205, 1975.

7. Saoud MFA. The infectivity and pathogenicity of geographical strains of Schistosoma mansoni. Transactions of the Royal Society of Tropical Medicine and Hygiene 60: 585-600, 1966.

8. Snedecor GW, Cochran WG. Statistical Methods $6^{\text {th }}$ edition lowa. lowa University Press.1977.

9. Vandewaa E.Bennett JI. HMG-CoA reductase: role in schistosoma egg production. In: Agabian N (ed) Molecular Strategies of Parasite Invasion, New York Liss. p. 627-640, 1987.

10. Vandewaa EA, Mills G, Foster LA, Bennett JL. Physiological role of HGM-CoA reductase in regulating egg production by Schistosoma mansoni. American Journal of Physiology. 257: 618-625, 1989. 